\title{
A MULTICHANNEL APPROACH TO METRIC-BASED SAR AUTOFOCUS
}

\author{
Robert L. Morrison, Jr. and Minh N. Do \\ Department of Electrical and Computer Engineering \\ Coordinated Science Laboratory \\ University of Illinois at Urbana-Champaign \\ rlmorris@uiuc.edu, minhdo@uiuc.edu
}

\begin{abstract}
The autofocus problem in synthetic aperture radar (SAR) is considered. We precisely characterize the multichannel nature of the SAR autofocus problem by constructing a low-dimensional subspace where the perfectly-focused image resides. To obtain a unique solution, we perform a sharpness optimization within this subspace. The subspace characterization enables the sharpness optimization to be performed in a vector space, which is conceptually simpler, and allows the SAR autofocus problem to be cast into a similar framework with other image restoration problems where fast algorithms and efficient methods have been established. We present experimental results demonstrating that the proposed framework can be used to bring the ideas of blind multichannel deconvolution techniques to the SAR autofocus problem, allowing the dimension of the solution subspace to be reduced further.
\end{abstract}

\section{INTRODUCTION}

Range measurement errors and atmospheric turbulence cause undesired phase shifts in the acquired SAR imaging data. The result is that the Fourier transform of the SAR scene is corrupted by multiplicative phase errors, so that the produced imagery is defocused. A mathematical model relating the perfectly-focused SAR data $G(k, n)$ to the defocused data $\tilde{G}(k, n)$ is expressed as [1]:

$$
\tilde{G}(k, n)=G(k, n) e^{j \phi_{e}(k)},
$$

where $k$ is a discrete frequency index in the cross-range dimension, $n$ is a discrete spatial-domain index in the range dimension, and $\phi_{e}(k)$ is a 1-D Fourier phase error function varying in cross-range only. The SAR image $\tilde{g}$ is formed by applying a 1-D inverse DFT to $\tilde{G}$ with respect to $k: \tilde{g}(m, n)=D F T_{k}^{-1}\{\tilde{G}(k, n)\}$, where $1 \leq m \leq M, 1 \leq n \leq N$. The result in the spatial-domain is that each column of the perfectly-focused image is defocused by a common blurring kernel $b(m)$ :

$$
\tilde{g}(m, n)=g(m, n) \circledast{ }_{M} b(m),
$$

where $\circledast_{M}$ denotes $M$-point circular convolution, and $b(m)=D F T_{k}^{-1}\left\{e^{j \phi_{e}(k)}\right\}$. The aim of SAR autofocus is to form an estimate of the perfectly-focused image $\hat{g}$ from the defocused image $\tilde{g}$ and prior assumptions about the SAR imaging scenario.

From the above description, we see that there is a multichannel nature to the SAR autofocus problem. Figure 1 presents this analogy; the columns of the perfectly-focused image $g$ can be viewed

This work was supported by the National Science Foundation under Grant CCR 0430877.

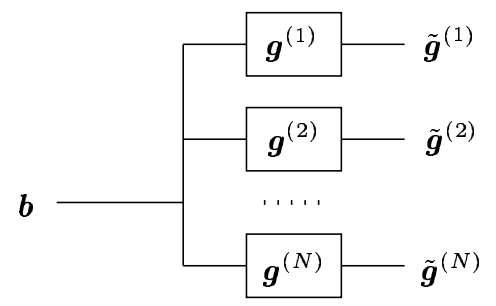

Fig. 1. Diagram illustrating the multichannel nature of the autofocus problem. Here, $\boldsymbol{b}$ is the blurring kernel, $\left\{\boldsymbol{g}^{(n)}\right\}$ are the perfectly-focused image columns, and $\left\{\tilde{\boldsymbol{g}}^{(n)}\right\}$ are the defocused columns.

as a bank of parallel filters that are excited by a common input signal, which is the blurring kernel $b$. In this paper, we devise a framework that explicitly captures the multichannel nature of the SAR autofocus problem via the construction of a subspace that characterizes the perfectly-focused solution. More specifically, given a defocused image $\tilde{g}$, we can precisely characterize the $M \times N$ pixel original image to live in an $M$-dimensional subspace. A unique solution can be obtained via a sharpness optimization within this restricted search space.

The multichannel framework allows us to derive and understand existing autofocus algorithms, and potentially leads to new algorithms offering improved restoration quality and computational performance. For example, this framework provides a link between blind multichannel deconvolution techniques, which are used in communications and other imaging problems, and the SAR autofocus problem [2]. This allows an approximate basis for the solution to be constructed with a much smaller dimension. We note that although the multichannel condition of the SAR autofocus problem has never been explicitly characterized as we do in this paper, the success of all existing autofocus approaches relies on this implicit assumption [1], [3].

\section{THE MULTICHANNEL-BASED FRAMEWORK}

In this section, we establish the multichannel framework for the SAR autofocus problem. We first define a basis, where $M$ coefficients associated with the basis parameterize the perfectly-focused solution. With a basis constructed for the solution subspace, an optimization problem can be formulated. The optimal coefficients 
are determined by minimizing an image cost metric (maximizing sharpness) using a gradient-descent algorithm [3].

In the following development, we will represent the perfectly-focused and defocused images by the $M$ by $N$ matrices $\boldsymbol{g}$ and $\tilde{\boldsymbol{g}}$, respectively; we will utilize the convention that the matrix rows correspond to the cross-range coordinate, while the columns correspond to the range coordinate. The unknown focused image is related to the given defocused image by a circular convolution with an unknown all-pass filter $\boldsymbol{f}$ (the inverse of the blurring filter b) that is applied to each column. The circular convolution can be described by multiplication with the $M$ by $M$ circulant matrix $\boldsymbol{C}\{\boldsymbol{f}\}$ :

$$
\boldsymbol{g}=\boldsymbol{C}\{\boldsymbol{f}\} \tilde{\boldsymbol{g}}
$$

Writing

$$
\boldsymbol{f}=\sum_{m=1}^{M} f_{m} \boldsymbol{e}_{m},
$$

where $\left\{\boldsymbol{e}_{m}\right\}_{m=1}^{M}$ is a basis for $\mathbb{C}^{M}$ and using the linear property of circular convolution, we have

$$
\boldsymbol{C}\{\boldsymbol{f}\}=\sum_{m=1}^{M} f_{m} \boldsymbol{C}\left\{\boldsymbol{e}_{m}\right\}
$$

From this, the unknown perfectly-focused image $\boldsymbol{g}$ can be expressed in terms of a basis expansion as

$$
\boldsymbol{g}=\sum_{m=1}^{M} f_{m} \boldsymbol{\varphi}^{(m)}
$$

where

$$
\boldsymbol{\varphi}^{(m)}=\boldsymbol{C}\left\{\boldsymbol{e}_{m}\right\} \tilde{\boldsymbol{g}}
$$

are basis vectors forming an $M$-dimensional subspace that contains the solution. This representation could be beneficial when we know $\boldsymbol{f}$ can be described by a small number of parameters (i.e., $\boldsymbol{f}$ has only a few non-zero coefficients). In this case, the dimension of the subspace where the perfectly-focused image resides can be reduced.

Note that $\boldsymbol{f}$ is the unit-impulse response of an all-pass filter. This prior information can also be directly captured in a basis expansion as follows. Since $\boldsymbol{C}\{\boldsymbol{f}\}$ is circulant, it is diagonalized by the $M$ by $M$ DFT matrix $\boldsymbol{W}$, and thus we can rewrite (1) as

$$
\boldsymbol{g}=\boldsymbol{W}^{H} \boldsymbol{\Lambda}_{\boldsymbol{\lambda}} \boldsymbol{W} \tilde{\boldsymbol{g}}
$$

where $\boldsymbol{\Lambda}_{\boldsymbol{\lambda}}$ is an $M$ by $M$ diagonal matrix with entries $\boldsymbol{\lambda}=D F T\{\boldsymbol{f}\}$ along the diagonal. Since $\boldsymbol{f}$ is all-pass, we can write $\lambda_{m}=e^{j \phi_{m}}$. Decomposing $\boldsymbol{\Lambda}_{\boldsymbol{\lambda}}$ as

$$
\boldsymbol{\Lambda}_{\boldsymbol{\lambda}}=\sum_{m=1}^{M} e^{j \phi_{m}} \boldsymbol{\Lambda}_{m}
$$

where $\boldsymbol{\Lambda}_{m}$ is a diagonal matrix with a one on the $m$-th element of the diagonal and zero elsewhere, we obtain

$$
\boldsymbol{g}=\sum_{m=1}^{M} e^{j \phi_{m}} \boldsymbol{\psi}^{(m)}
$$

where

$$
\boldsymbol{\psi}^{(m)}=\boldsymbol{W}^{H} \boldsymbol{\Lambda}_{m} \boldsymbol{W} \tilde{\boldsymbol{g}}
$$

The fixed vectors $\left\{\boldsymbol{\psi}^{(m)}\right\}_{m=1}^{M}$ form an $M$-dimensional basis, where the phases $\phi_{m}$ parameterize the solution. It is easy to verify that (5) provides a complete characterization to all possible original images given the blurred image.

The autofocus problem can now be cast as to find an image satisfying (5) in addition with other conditions (e.g., minimum entropy or sparsest solution). As an example, we will consider the representation in (5), and examine the problem of determining the $M$ phases $\phi_{m}$ that maximize the image sharpness. Specifically, we seek a solution to the following optimization problem:

$$
\hat{\boldsymbol{\phi}}=\arg \min _{\phi} C(\boldsymbol{I}(\boldsymbol{\phi})),
$$

where

$$
C(\boldsymbol{I})=\sum_{p=1}^{M N} c\left(I_{p}\right),
$$

$c(\cdot)$ is a sharpness metric operating on the intensity of each pixel $I_{p}=\left|g_{p}\right|^{2}$, and $p$ is the pixel index, $1 \leq p \leq M N$.

\section{DERIVATION OF GRADIENT-BASED SEARCH}

We provide an example showing how the existing gradient-based approach, proposed in Fienup et al. [3], can be derived from our framework. The optimization problem may be solved using a gradient-descent method. In the $(l+1)$-th iteration, the phases are updated according to:

$$
\phi^{(l+1)}=\phi^{(l)}-\alpha_{l} \nabla_{\phi} C\left(\phi^{(l)}\right),
$$

where $\phi^{(l)}$ denotes the phase estimate in the $l$-th iteration, $\alpha_{l}$ is a step size parameter, and $\nabla_{\phi} C(\phi)$ is the gradient of the metric objective function in (7) with respect to $\phi$ :

$$
\nabla_{\phi} C(\phi)=\left[\frac{\partial C}{\partial \phi_{1}}, \frac{\partial C}{\partial \phi_{2}}, \ldots, \frac{\partial C}{\partial \phi_{M}}\right]^{T} .
$$

The convergence rate of the gradient-descent algorithm depends on how the parameter $\alpha_{l}$ is selected; in our implementation, $\alpha_{l}$ is determined through a 1-D line search using the Armijo rule [4].

The gradient (10) for images parameterized by (5) and an arbitrary metric of the form (8) is derived as follows. First, the derivative with respect to a single phase $\phi_{k}, 1 \leq k \leq M$, is determined as

$$
\frac{\partial C}{\partial \phi_{k}}=\sum_{p=1}^{M N} \frac{\partial c\left(I_{p}\right)}{\partial I_{p}} \frac{\partial I_{p}}{\partial \phi_{k}} .
$$

Using the basis expansion for the images in the search space (5), we can express each pixel intensity as

$$
\begin{aligned}
I_{p} & =g_{p} g_{p}^{*} \\
& =\left(\sum_{m=1}^{M} e^{j \phi_{m}} \psi_{p}^{(m)}\right)\left(\sum_{m=1}^{M} e^{-j \phi_{m}}\left(\psi_{p}^{(m)}\right)^{*}\right),
\end{aligned}
$$

where $*$ denotes complex conjugate. From this, we obtain

$$
\begin{aligned}
\frac{\partial I_{p}}{\partial \phi_{k}} & =-j g_{p}\left(\psi_{p}^{(k)}\right)^{*} e^{-j \phi_{k}}+j g_{p}^{*} \psi_{p}^{(k)} e^{j \phi_{k}} \\
& =-2 \Im\left\{\left(\sum_{m=1}^{M} e^{j \phi_{m}} \psi_{p}^{(m)}\right)^{*} \psi_{p}^{(k)} e^{j \phi_{k}}\right\},
\end{aligned}
$$




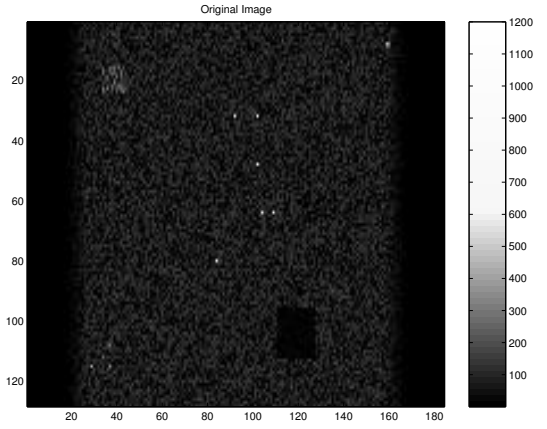

(a)

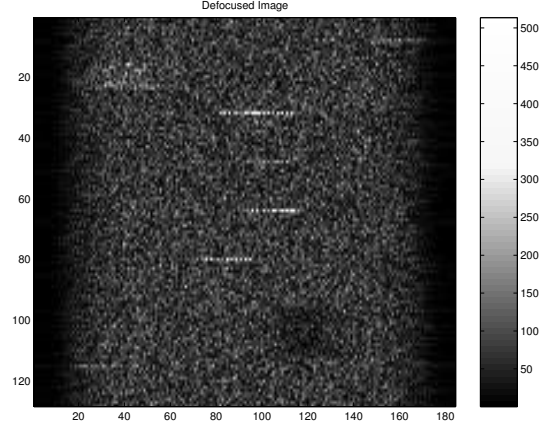

(b)

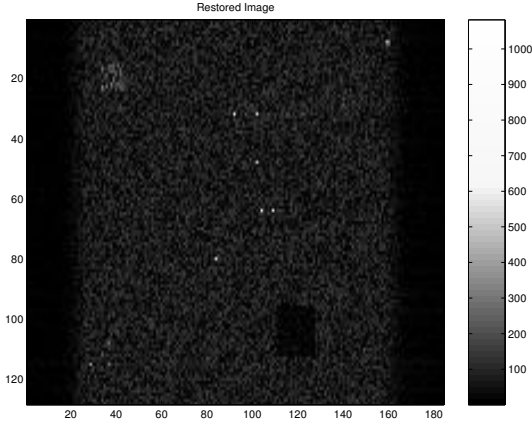

(c)

Fig. 2. The magnitude of a complex-valued 184 by 128 pixel synthetic SAR image: (a) perfectly-focused image, (b) defocused image produced by applying an eighth-order polynomial phase error, and (c) restoration formed through entropy minimization on the approximate basis. Note that the images are displayed transposed; the rows correspond to the range coordinate, and the columns to the cross-range.

where $\Im\{\cdot\}$ specifies the imaginary part of the argument. In this case, for the basis representation in (5-6), the derivative formula in (12) turns out to be the same as the formula derived by Fienup et al. [3] in the Fourier domain. Through this connection, the gradient (10) can be efficiently computed using the FFT and requires $O(M N \log M)$ operations [3].

The derivative of $c\left(I_{p}\right)$ with respect to $I_{p}$ in (11) is specific to the selected metric. As an example, we consider the entropy metric

$$
c_{H}\left(I_{p}\right)=-I_{p} \ln \left(I_{p}\right)
$$

which has the derivative

$$
\frac{\partial c_{H}\left(I_{p}\right)}{\partial I_{p}}=1+\ln \left(I_{p}\right)
$$

\section{APPLICATION OF THE MULTICHANNEL FRAMEWORK}

Using the multichannel framework for the SAR autofocus problem, we determined that the $M \times N$ perfectly-focused image can be represented using an $M$-dimensional basis. By approximating the circular convolution in the SAR autofocus problem with a linear convolution, and exploiting the fact that SAR images are dominated by sparse, point-like features, an approximate basis for the perfectly-focused image can be formed with dimension $K \ll M$. In other words, we can further reduce the dimension of the search space for the perfectly-focused image.

When the filtering operation in Figure 1 is described by linear convolution, it is possible (under certain conditions) to construct a matrix operator $\boldsymbol{A}$ formed from the output signals $\left\{\tilde{\boldsymbol{g}}^{(n)}\right\}$ such that the nullspace of $\boldsymbol{A}$ is a scaled version of the underlying filter responses $\left\{\boldsymbol{g}^{(n)}\right\}$. This idea underlies the EVAM (or eigenvectorbased) approach for blind multichannel deconvolution, which has applications in communications and other imaging problems [2]. The approach requires the $z$-transforms of the filters to be coprime, and the common input signal $\boldsymbol{b}$ to be persistently exciting. We motivate this approach using the simplest example of a twochannel system. Conceptually, the idea is to pass the blurred signals $\left\{\tilde{\boldsymbol{g}}^{(1)}, \tilde{\boldsymbol{g}}^{(2)}\right\}$ through another bank of parallel filters $\left\{\boldsymbol{u}^{(1)}, \boldsymbol{u}^{(2)}\right\}$. The goal is to determine the filter responses $\boldsymbol{u}^{(1)}$ and $\boldsymbol{u}^{(2)}$ such that the sum of the output signals is zero:

$$
\tilde{g}^{(1)}(m) * u^{(1)}(m)+\tilde{g}^{(2)}(m) * u^{(2)}(m)=0,
$$

$m=1,2, \ldots, L+R-1$, where $L$ is the length of $\tilde{\boldsymbol{g}}^{(n)}$, and $R$ is the length of $\boldsymbol{g}^{(n)}$ and $\boldsymbol{u}^{(n)}, n=1,2$. Given the required conditions hold, and from $\tilde{g}^{(n)}(m)=g^{(n)}(m) * b(m)$, it can be shown that $\boldsymbol{u}^{(1)}$ and $\boldsymbol{u}^{(2)}$ must be scaled versions of $\boldsymbol{g}^{(2)}$ and $\boldsymbol{g}^{(1)}$, respectively: $u^{(1)}(m)=\alpha g^{(2)}(m)$ and $u^{(2)}(m)=-\alpha g^{(1)}(m)$ for any constant $\alpha$ [5]. The EVAM matrix operator for this case is constructed as

$$
\boldsymbol{A}=\left[\boldsymbol{C}^{*}\left\{\tilde{\boldsymbol{g}}^{(2)}\right\} \mid-\boldsymbol{C}^{*}\left\{\tilde{\boldsymbol{g}}^{(1)}\right\}\right],
$$

where $\boldsymbol{C}^{*}\left\{\tilde{\boldsymbol{g}}^{(n)}\right\}$ is the $(L+R-1) \times R$ convolution matrix for the signal $\tilde{\boldsymbol{g}}^{(n)}$. From the arguments above, we see that the nullspace of $\boldsymbol{A}$ is spanned by the vector $\boldsymbol{v}=\alpha\left[\boldsymbol{g}^{(1) T} \mid \boldsymbol{g}^{(2) T}\right]^{T}$. When an arbitrary number of channels is considered, $\boldsymbol{A}$ is constructed so that (14) is evaluated for every pair of channel responses $\boldsymbol{g}^{(n)}$ and $\boldsymbol{g}^{(l)}, n \neq l$.

A link between the eigenvector-based deconvolution technique and the SAR autofocus problem is established by using the linear convolution framework to obtain an approximate solution to the circular deconvolution problem. Given prior knowledge that the underlying image is spatially limited (e.g., there are a sufficient number of zero rows), the approximation to linear convolution becomes accurate. In this case, the EVAM operator $\boldsymbol{A}$ formed from the defocused image columns has full column rank (since the convolution is not exactly linear). However, the smallest singular values of $\boldsymbol{A}$ are close to zero, so that the corresponding right singular vectors form a space of solutions $\left\{\boldsymbol{v}_{i}\right\}_{i=1}^{K}$ where $\left\|\boldsymbol{A} \boldsymbol{v}_{i}\right\|_{2}$ is approximately zero. The perfectly-focused image lies within this space (to a good approximation), and may be recovered by optimizing a particular metric.

Figure 2(a) shows a perfectly-focused simulated 184 by 128 pixel SAR image that has been spatially limited to 128 by 128 pixels through the application of a raised cosine window. Such a scenario is of practical interest when the Fourier data is oversampled in the cross-range dimension (i.e., through increasing the radar pulse repetition rate) so that the image coverage extends beyond the antenna footprint. A defocused image, formed by applying an eighth-order polynomial phase error, is displayed in Figure 


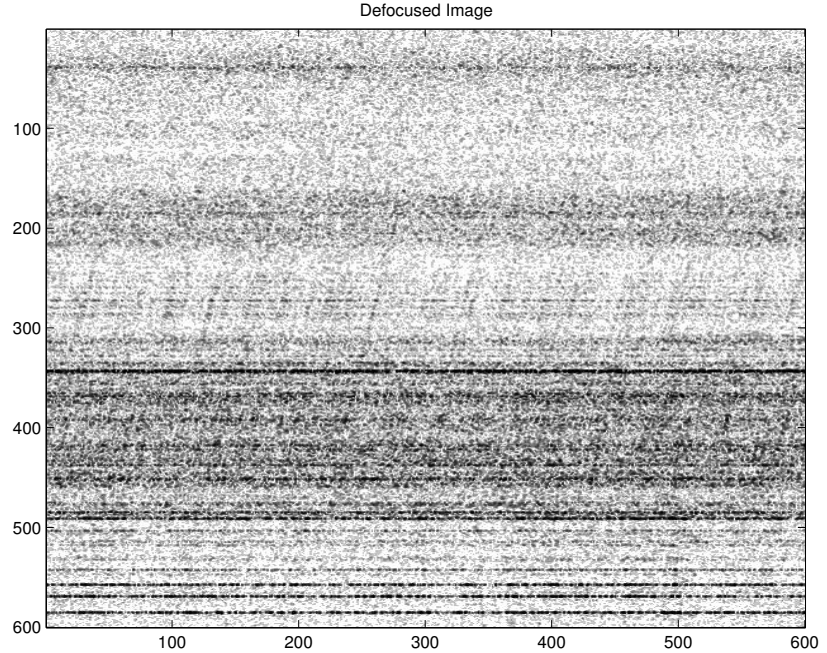

(a)

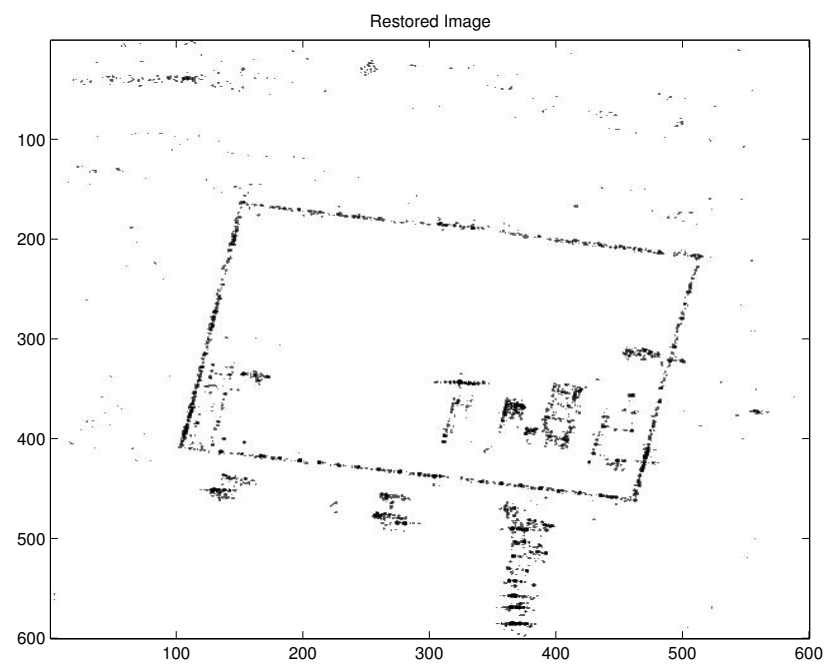

(b)

Fig. 3. Restoration of an actual SAR image where a white Fourier phase error has been applied: (a) defocused image and (b) restoration formed using entropy on the approximate basis. Note that the images are displayed transposed with respect to the text.

2(b). Forming the EVAM matrix using every column of the defocused image is computationally infeasible, so therefore we select three image columns with the largest energy to construct $\boldsymbol{A}$. The approximate basis for the solution $\left\{\boldsymbol{v}_{i}\right\}$ is determined by taking the SVD of $\boldsymbol{A}$ and using the right singular vectors corresponding to the smallest singular values. The entropy metric is used within the basis to determine an estimate of the perfectly-focused image columns. By applying the conjugate of the restored image columns (zero-padded to the length of the image) to the corresponding defocused image columns, an estimate of the phase error is obtained. Figure 2(c) shows the restored image formed by applying the phase error estimate. In this example, the optimization is performed over 13 vectors, a reduction from the space of 184 vectors that characterizes the perfectly-focused image exactly. The number of vectors used in the optimization is determined by visual inspection of the singular values; the right singular vectors of $\boldsymbol{A}$ are selected that correspond to singular values close to zero.

Figure 3(a) shows an 600 by 600 pixel actual SAR image that has been defocused using a white phase error (uniformly distributed between $-\pi$ and $\pi$ and independent between frequency components). The EVAM operator $\boldsymbol{A}$ is formed using three columns of the defocused image truncated to 121 pixels about the center of the image; here, prior knowledge that dominant scatterers exist near the center of the image has been utilized, and we assume that the scatterers are spatially limited to a region of 45 pixels in the cross-range dimension (i.e., $L=121$ and $R=45$ ). Figure 3(b) shows the restoration formed using the same procedure as the experiment of Figure 2. Although the white phase error produces a blurring response that is not spatially limited, the entropy metric is able to pick out the prominent point-like features in the underlying image. The optimization is performed over a space of 80 vectors, which is determined through inspection of the singular values of $\boldsymbol{A}$. Because of the severity of the blurring operator, none of the singular values are close to zero; in this experiment, we select the right singular vectors that correspond to singular values less than fifteen percent of the largest singular value of $\boldsymbol{A}$.

\section{CONCLUSION}

In this paper we have presented a multichannel framework for the SAR autofocus problem. The novelty of the approach is that the multichannel nature of the SAR autofocus problem is utilized explicitly to characterize a subspace for the perfectly-focused image. Sharpness optimization techniques may be used to determine a unique restoration within the subspace. Experimental results demonstrate how the proposed framework provides a link to blind multichannel deconvolution techniques, enabling the construction of an approximate basis for the solution with reduced dimension.

Acknowledgment. The authors would like to thank Dr. Charles Jakowatz, Jr. of Sandia National Laboratories for supplying the actual SAR image presented in this paper.

\section{REFERENCES}

[1] C. V. Jakowatz, Jr., D. E. Wahl, P. H. Eichel, D. C. Ghiglia, and P. A. Thompson, Spotlight-Mode Synthetic Aperture Radar: A Signal Processing Approach., Kluwer Academic Publishers, Boston, 1996.

[2] M. Gurelli and C. Nikias, "EVAM: An eigenvector-based algorithm for multichannel blind deconvolution of input colored signals," IEEE Trans. on Signal Processing, vol. 43, no. 1, pp. 134-149, January 1995.

[3] J. R. Fienup and J. J. Miller, "Aberration correction by maximizing generalized sharpness metrics," Journal of the Optical Society of America A, vol. 20, no. 4, pp. 609-620, April 2003.

[4] C. T. Kelley, Iterative Methods for Optimization., Society for Applied and Industrial Mathematics, Philadelphia, 1999.

[5] G. Harikumar and Y. Bresler, "Blind restoration of images blurred by multiple filters: Theory and efficient algorithms," IEEE Trans. on Image Processing, vol. 8, no. 2, pp. 202-219, February 1999. 